\title{
Ductoscopy-guided and Conventional Surgical Excision
}

a report by

Seema $A$ Khan, MD

Department of Surgery Feinberg School of Medicine and Robert H Lurie Comprehensive Cancer Center of

Northwestern University

DOI: 10.17925/OHR.2006.00.00.1i

Radiologic imaging is routinely used to evaluate women with spontaneous nipple discharge (SND), but definitive diagnosis is usually only achieved by surgical terminal duct excision (TDE). Ductoscopy has been reported to result in improved localization of intraductal lesions and may avoid surgery in women with endoscopically normal ducts.

Nipple discharge is responsible for approximately $5 \%$ of annual surgical referrals. ${ }^{1}$ Not all forms of spontaneous nipple discharge (SND) are associated with significant pathologic findings. The clinical features of SND that are associated with a high likelihood of intraductal neoplasia include unilaterality, persistence, emanation from a single duct, and watery, serous, or bloody appearance. ${ }^{2,3}$ Discharges with these characteristics are classified as pathologic and have traditionally been considered an indication for surgical excision of the involved duct. However, among women with SND who are evaluated surgically, 2-25\% are diagnosed with either invasive or non-invasive cancer..$^{2-5}$

When mammography shows significant findings in women with SND, the likelihood of malignancy increases, ${ }^{2}$ but mammographic imaging is often unhelpful. Galactography has been used to evaluate women with SND with variable success. ${ }^{6,7}$ When SND is caused by peripheral intraductal lesions, galactography provides localizing information and can also assess the likelihood of malignancy, ${ }^{4}$ although definitive diagnosis requires central or terminal duct excision (TDE). Duct excision is also therapeutic unless malignancy is discovered. ${ }^{2,8}$ Mammary endoscopy (ductoscopy) is a recently introduced technique that may allow more precise identification and delineation of intraductal disease but is not currently a standard practice among most surgeons. Ductoscopy has been reported to result in improved localization of intraductal lesions ${ }^{9-11}$ and may avoid surgery in women with endoscopically normal ducts. However, ductoscopy adds to time and expense in the operating room (OR), and the yield of significant pathologic lesions reported in separate series of women who are managed with and without ductoscopy at different institutions is reported to be in the range of $90 \% .^{2,9}$ There has not been a direct comparison of the results achieved with these two different approaches in terms of lesion yield or amount of tissue excised. The authors undertook a comparison of findings in women with SND who were managed with and without ductoscopy

1. Dixon J M, Mansel R E, "ABC of breast diseases. Symptoms assessment and guidelines for referral”, BMJ (1994);309: pp. 722-726.

2. Cabioglu N, Hunt K K, Singletary S E et al., "Surgical decision making and factors determining a diagnosis of breast carcinoma in women presenting with nipple discharge”, J. Am. Coll. Surg. (2003);196: pp. 354-364.

3. King T A, Carter K M, Bolton J S et al., "A simple approach to nipple discharge", Am. Surg. (2000);66: pp. 960-965.

4. Hou M F, Huang T J, Liu G C, "The diagnostic value of galactography in patients with nipple discharge", Clin. Imaging. (2001);25: pp. 75-81.

5. Wong L, Chung Y F, Wong CY, "Microdochectomy for single-duct nipple discharge", Ann. Acad. Med. Singapore (2000);29: pp. 198-200.

6. Dawes L G, Bowen C, Venta L A et al., "Ductography for nipple discharge: no replacement for ductal excision", Surgery (1998);124: pp. 685-691.

7. Van Zee KJ, Ortega P G, Minnard E et al., "Preoperative galactography increases the diagnostic yield of major duct excision for nipple discharge”, Cancer (1998);82: pp. 1,874-1,880.

8. Gioffre' Florio M A, Manganero T, Pollicino A et al., "Surgical approach to nipple discharge: a ten year experience", J. Surg. Oncol. (1999); 71: pp. 235-238.

9. Dietz J R, Crowe J P, Grundfest $S$ et al., "Directed duct excision by using mammary ductoscopy in patients with pathologic nipple discharge”, Surgery (2002);132: pp. 582-587.

10. Matsunaga T, Ohta D, Misaka T et al., "Mammary ductoscopy for diagnosis and treatment of intraductal lesions of the breast", Breast Cancer (2001);8: pp. 213-221.

11. Shen KW,Wu J, Lu J S et al., "Fiberoptic ductoscopy for patients with nipple discharge", Cancer (2000);89: pp. 1,512-1,519. 
to assess the value of adding ductoscopy to the management of these patients.

Patients underwent evaluation and treatment of SND, judged to be suggestive of intraductal neoplasia by clinical characteristics. Mammographic findings considered abnormal were clustered calcifications, densities that persisted with spot compressions, and dilated ducts. Ultrasound findings relevant to evaluation of nipple discharge were intraductal nodules and dilated ducts. For those patients who had successfully completed ductograms, significant findings included filling defects and tapered or narrowed ducts.

Both conventional TDE and ductoscopy-guided excision was performed in the OR, under local anesthesia, with sedation. For 15 women in the conventional surgery group, the TDE was preceded by a ductogram on the morning of surgery. In the remaining 43 women, TDE was performed using a peri-areolar incision centered on the discharging duct.

For the ductoscopy procedure, the discharging duct was identified and cannulated with a series of graded lacrimal probes, the $0.9 \mathrm{~mm}$ Acueity endoscope (Larkspur, California) was introduced and advanced under direct vision, and all tiers of branches examined until the endoscope could not be advanced further or an obstructing lesion was identified. If a lesion was found, the extent of disease was marked out on the skin by transillumination at the most proximal and most distal lesions, and the axial extent was marked when disease was present in multiple peripheral branches of the same ductal tree.

Until April 2001, all patients underwent conventional excision without ductoscopy, with pre-operative evaluation and the precise surgical approach depending on surgeon preference. The records of all women were reviewed for age, race, fluid characteristics, imaging studies, ductoscopy findings, histologic findings, presence or absence of cytologic atypia, and complications.

Results

One hundred and seventeen breasts from 114 women were evaluated for nipple discharge or abnormal ductal lavage cytology over an eight-year period. The patients' ages ranged from 25 to 83 with a mean of 50.5 years. The study population was divided almost evenly between those who had surgery alone (58) and those with ductoscopic guidance (59). Ductoscopy was attempted in 68 breasts and was unsuccessful in nine cases, either related to duct perforation (eight breasts; $11.8 \%$ ) or duct stricture (one breast; 1.4\%). These women proceeded to duct excision without endoscopic guidance at the same procedure and have been included in the conventional surgery group for purposes of analysis, although their results are also presented separately. Of the 59 breasts that were endoscoped, 49 (83\%) had one duct examined and $10(17 \%)$ had more than one duct examined.

Ultrasound was performed somewhat more frequently in the ductoscopy group, but the distribution of abnormal ultrasound findings is not different between the groups. Ductograms were successfully completed in 47 of 58 attempts (81\%). Results were normal in nine (19.1\%), filling defects were found in 35 (74.5\%), and dilated ducts in four (8.5\%).

Overall, non-proliferative benign change was found in 18 women $(15.4 \%)$ and hyperplasia of the usual type (HUT) in $10(8.6 \%)$. The most common intraductal neoplastic finding was papilloma in 73 patients (62.4\%). Atypical hyperplasia was present in six specimens $(5.1 \%)$, and duct carcinoma in situ (DCIS) was found in 10 (8.6\%). The yield of pathologic findings explaining the discharge was not significantly different between patients undergoing conventional or endoscopically guided resection, although overall yield was slightly higher in the ductoscopy group. This was true if hyperplasia without atypia was included as an explanation for the discharge and also if only findings of papilloma, atypia hyperplasia, or ductal carcinoma in situ were considered.

The endoscopic findings correlated well with the pathologic diagnoses; a papilloma or more severe lesion was found in 44 of 49 (90\%) ducts where an intraductal neoplasm was seen on ductoscopy. Of the 49 papillomatous lesions visualized, $36(73.5 \%)$ were indeed papilloma, five $(10.2 \%)$ were cancer, three $(6.1 \%)$ were atypical hyperplasia, and three were HUT. Pathologically, there were 37 papillomata diagnosed and 36 (97.3\%) of these were correctly identified at the time of ductoscopic evaluation. The authors observed a significant correlation between endoscopically visualized disease and the presence of intraductal neoplasia. The visualization of neoplastic lesions (papilloma or more severe) was not as good with imaging studies as with ductoscopy.

The maximal distance of visualized lesions from the nipple on ductoscopy was $10 \mathrm{~cm}$ (mean distance $4 \mathrm{~cm}$ ), whereas with ductography it was $5 \mathrm{~cm}$. Among the ductoscopy group, in 22 (37\%) women, the most centrally located abnormality was $5 \mathrm{~cm}$ or more from the nipple, and six of the 11 severe lesions (AH or DCIS) were included in this group. The number of lesions visualized was greater on ductoscopy than ductography.

Four cancers were found in the conventional surgery group and six in the ductoscopy group. Three of the cancers diagnosed in women undergoing conventional excision presented with diffuse mammographic calcifications (two) or a large palpable mass (one) in 
addition to SND. Of the six women who had intraoperative ductoscopy, five $(83.3 \%)$ had SND as the only presenting symptom and did not have corresponding radiologic or physical findings.

\section{Discussion}

Nipple discharge is the presenting breast complaint for less than $10 \%$ of women at any age presenting to breast practices. $^{8,12}$ Of those who have discharge, $35-65 \%$ are considered clinically benign or physiological and do not require surgical intervention. ${ }^{2,3,8}$ Surgical evaluation of patients with discharge suggestive of intraductal neoplasia renders a diagnosis of cancer in only $2-25 \%$ of cases.

Ductoscopy has recently emerged as a method of identifying intraductal neoplasia, and intraductal diagnostic biopsy techniques are evolving. There have been several published reports regarding the ability to visualize papillomata and other changes, but there has been no attempt to compare the yield of ductoscopyguided resection with that of conventional approaches using ductography, blue dye, or probe-guided resection for the diagnosis of intraductal lesions that cause SND. The authors have undertaken such an analysis because adding ductoscopy to the evaluation of these patients results in added cost in terms of equipment, supplies, and OR time. The authors report here the first analysis comparing results based on treatment with and without the use of ductoscopy. The authors found that the range of pathologic diagnoses for both groups was similar, although there is a non-significant trend toward a higher yield of ductal neoplasia with the use of ductoscopy compared with women who had conventional surgery (overall yield $88 \%$ versus $81 \%$ ).

The authors have found that ductoscopy is a useful adjunct to the evaluation of SND because they were able to identify multiple papillomata significantly more frequently (mean 1.7 with ductoscopy versus 1.0 with conventional surgery); they identified peripheral disease in a higher proportion of women, and found a higher proportion of atypical ductal hyperplasia with ductoscopy.

The authors have observed a trend toward the identification of more severe intraductal disease, which may merit further therapy (atypical hyperplasia or DCIS) with ductoscopy than with conventional surgery. In the ductoscopy group, 11 of 59 (18.6\%) women were found to have these more severe lesions. In the conventional surgery group, there were five of 58 such women (8.6\%). Lesions that have a larger impact on breast cancer risk (peripheral papillomata, multiple papillomata, and atypical hyperplasia) were therefore found more often with ductoscopy-guided excision than with conventional surgery

The authors identified more peripheral lesions and more multiple papillomata using ductoscopy than was possible in the conventional surgery group. Owing to the fact that both peripheral location and multiplicity of papillomata has been associated with increased risk of breast cancer, ${ }^{8}$ the ability to identify these features of papillary neoplasia may be significant in terms of risk assessment. The association of atypical hyperplasia with papillomata increases breast cancer risk to the same extent as atypical hyperplasia alone, and the subsequent carcinomas have been reported to arise in the same location as the original papilloma. ${ }^{13}$ Complete resection of disease around papillomata may therefore have significance in terms of breast cancer risk assessment and use of preventive interventions.

\section{Conclusions}

Ductoscopy is an emerging technology, not yet widely adopted in the US. Other applications of ductoscopy that have been proposed include the delineation of intraductal disease in women undergoing breastconserving surgery for cancer ${ }^{14}$ and the investigation of women with atypical cytologic findings on ductal lavage. ${ }^{15,16}$ The use of these has yet to be established, and as greater experience accumulates with this tool, issues that need to be addressed include the cost of the procedure as well as the biologic significance of the additional disease that is identified by using it.

The development of ablative methods and methods to distinguish benign from malignant ductal lesions may afford the possibility of safely ruling out the need for surgical intervention in select cases in the future. A large-scale prospective study with expanded indications is needed to demonstrate the potential for quick, facile but effective intraductal evaluation.

12. Hughes L E, Mansel R E, Webster D J, Benign Disorders and Diseases of the Breast: Concepts and Clinical Management, 2nd ed. (2000).

13. Page D L, Salhany K E, Jensen $R A$ et al., "Subsequent breast carcinoma risk after biopsy with atypia in a breast papilloma", Cancer (1996); 78: pp. 258-266.

14. Dooley W C, "Routine operative breast endoscopy during lumpectomy”, Ann. Surg. Oncol. (2003);10: pp. 38-42.

15. Khan S A, Baird C, Staradub V L et al., "Ductal lavage and ductoscopy: the opportunities and the limitations", Clin Breast Cancer (2002);3: pp. 185-191.

16. Dietz J R, Rastelli A, Bokern J et al., "Mammary ductoscopy to further characterize ductal lavage-diagnosed atypia: correlation between cytology, endoscopy, and surgical pathology”, abstract, Ann. Surg. Oncol. (2004);11(suppl.):S101-S102. 